\title{
Abatacept-based Graft-Versus-Host-Disease Prophylaxis in Haplo-identical Hematopoietic Cell Transplant: Single Center Experience in a High-Risk Cohort
}

\author{
Enass Raffa ${ }^{1}$, Anand Srinivasan ${ }^{1}$, Donna Wall ${ }^{2}$, Tal Schechter ${ }^{1}$, Muhammad Ali ${ }^{1}$, Joerg \\ Krueger $^{2}$, and Kuang-Yueh chiang ${ }^{2}$ \\ ${ }^{1}$ The Hospital for Sick Children \\ ${ }^{2}$ Hospital for Sick Children
}

June 25, 2021

\begin{abstract}
Post-transplant cyclophosphamide (PTCy) has become the most popular approach in haplo-identical hematopoietic cell transplant (haplo-HCT). Although there are reports of a small number of adult patients in the literature who experienced graft failure and were re-transplanted with a haploidentical donor with PTCy prophylaxis, there is still insufficient guidance for patients with specific contraindications/complications to cyclophosphamide and virtually no data in the pediatric setting. Abatacept (Aba), a $\mathrm{T}$ cell co-stimulation blockade, has been shown in previous studies to prevent severe acute graft-versus-host disease (GVHD) with minimal toxicity and durable engraftment. We report the efficacy of Aba-based GVHD prophylaxis in four pediatrics patients (ages 2-12 years) who received a haplo-HCT with peripheral blood stem cells (PBSC). Three patients had previous transplants. One patient developed acute GVHD of skin stage 3 and one patient had both stage 3 skin and stage 1 GI acute GVHD. Two patients had mild chronic skin GVHD. All 4 patients are alive with full donor chimerism and without disease at 7-23 months follow up, weaning or off immunosuppressive agents with no complications. Successful haplo-HCT utilizing an Ababased regimen can result in reliable engraftment and acceptable GVHD. However, our small sample size limits generalizability and encourages the consideration of a larger prospective trial to validate these results in the haplo-HCT setting.
\end{abstract}

\section{INTRODUCTION}

The platform of haplo-HCT has changed dramatically in recent decades, specifically with the introduction of post-transplant cyclophosphamide (PTCy) $(1,2)$. Several groups have since combined the use of PTCy and anti-thymocyte globulin (ATG), with varying doses, in an effort to further reduce GVHD with haplo-HCT when a PBSC graft is used (3-6). This regimen was shown to reduce the incidence of both acute and chronic GVHD, had lower NRM, with similar long term transplant outcomes. This highlights the possibility of preserving a good outcome post haplo-HCT with a variation in the standard use of PTCy alone.

Haplo-HCT with PTCy has been successfully used as salvage therapy in situations where the initial non-haplo HCT has failed. However, with the increasing popularity and success of haplo-HCT, we find ourselves facing unique challenges $(7,8)$. Although there are reports of a small number of adult patients in the literature who experienced graft failure or loss who were re-transplanted with a haploidentical donor with PTCy prophylaxis as well, there is still insufficient guidance for patients with specific contraindications/complications to cyclophosphamide and virtually no data in the pediatric setting (9-13). The concern regarding further use of cyclophosphamide may include, but is not limited to, recent exposure and significant cumulative dose of cyclophosphamide and presence of secondary organ toxicity such as cardiac dysfunction or urinary tract toxicity. Abatacept, a soluble fusion protein composed of the extracellular domain of human cytotoxic Tlymphocyte -associated antigen 4 (CTLA-4) linked to the modified Fc portion of human immunoglobulin 
G1, selectively inhibits T cell co-stimulation by blocking CD28 mediated signaling (14). It therefore can attenuate T cell activation giving it the potential to mitigate GVHD (15). Abatacept earned the breakthrough designation from the US Food and Drug Administration for protection against acute GVHD in 2019 (16, 17).

In this single center retrospective study, we describe the use of abatacept for GVHD prophylaxis in four patients. This is the first report to describe the use of abatacept as an alternative to the PTCy approach in haplo-HCT in the malignant setting. The standard PTCy regimen had been avoided in these patients either due to previous exposure to PTCy or when administration of PTCy would not be appropriate due to their underlying disease or organ dysfunction.

\section{METHODS}

We describe our experience with using abatacept (Aba) as GVHD prophylaxis in four pediatric patients who received haplo-HCT. Our study was approved by the institutional Research Ethics Board. We conducted a retrospective chart review on all children who received a haploidentical HCT at The Hospital for Sick Children (Sickkids) in Toronto, Canada between January 2015 and July 2020.

Inclusion criteria consisted of: (a) age $<18$ years at time of transplantation, (b) recipient of a peripheral blood stem cell graft from HLA haploidentical donors (c) received non PTCy GVHD prophylaxis, and (d) may have malignant or non-malignant disorder. All patients who met the inclusion criteria have had at least 180 day follow up post-transplant at the time of this report. There were five recipients who were eligible for inclusion in this cohort. One patient was excluded from analysis to avoid confounding the outcome as the patient had also received immunosuppression with etanercept as part of the treatment for underlying disease.

\section{SUPPORTIVE CARE}

All our patients were treated in protective private HEPA (high-efficiency-particulate-air) filtered positive pressure rooms. Infectious prophylaxis (antimicrobial, antiviral and antifungal) was administered as per our institutional guidelines. All patients received weekly monitoring of qualitative/quantitative polymerase chain reaction (PCR) for Epstein Barr virus (EBV), Adenovirus, CMV and Human herpes virus-6 (HHV-6). Treatment was guided by quantitative viral loads when positive. Since May 2020, all patients are required to have a COVID19 (Coronavirus disease 2019) nasopharyngeal PCR swab prior to admission, procedures or at the emergence of new symptoms suggestive of an infection. Acute GVHD was graded according to modified Glucksberg criteria and chronic GVHD was scored based on National Institutes of Health global severity criteria $(18,19)$. Chimerism studies were performed at the time of WBC recovery.

\section{RESULTS}

Between January 2015 and July 2020, 40 patients received a haploidentical transplant at The Hospital for Sick Children. Indications for transplant included: AML $(\mathrm{n}=11)$, ALL $(\mathrm{n}=9)$, immunodeficiency $(\mathrm{n}=4)$, marrow failure $(n=4)$, lymphoma $(n=3)$, chronic myeloid leukemia $(n=3)$, mixed phenotypic acute leukemia $(n=2)$, myelodysplastic syndrome $(n=2)$, and other $(n=2)$. From this cohort, five patients received a haploidentical transplant with a non PTCy approach. We excluded one of the five patients because that patient also received a non PTCy prophylaxis with etanercept.

Patient demographics and transplantation details are summarized in Table 1. A total of four patients (2-12 years) received a haploidentical HCT with a PBSC graft and Aba-based GVHD prophylaxis. Our cohort included one patient with a prior liver transplant for erythropoietic porphyria, two patients who have had a previous haplo-HCT with PTCy for hematological malignancy (CML and AML) complicated by secondary graft failure and one patient with a primary immunodeficiency (interferon gamma receptor 1 [IGR1] deficiency) who had concurrent mycobacterial avian complex infection.

All our cohort had significant concerns for the use of PTCy GVHD prophylaxis either due to recent prior high dose cyclophosphamide, concern of alkylating therapy exposure or organ dysfunction and therefore required 
an alternative regimen. Our GVHD prophylaxis included Aba $(10 \mathrm{mg} / \mathrm{kg})$ on day $-1 \&+5$ in four patients, methotrexate $\left(5 \mathrm{mg} / \mathrm{m}^{2}\right)$ on day $+1,3, \& 6$ in three patients, tacrolimus starting on day +1 , in four patients and mycophenolate mofetil starting on day +1 , in four patients. Two patients received two additional doses of abatacept (four total) on day +32 and day +56 for acute skin GVHD. There were no infusion reactions or adverse effects noted with Aba infusion. Rituximab $\left(375 \mathrm{mg} / \mathrm{m}^{2}\right)$ was given on day +1 as EBV prophylaxis in two patients with EBV seropositivity.

The median number of CD34 ${ }^{+}$cells dose was $6.75 \times 10^{6}$ cells $/ \mathrm{kg}$.

The median time to neutrophil and platelet $\left(>20 \times 10^{9} / \mathrm{L}\right)$ engraftment was 23 days (range, 20-26 days) and 51 days (range, 17-91 days), respectively. Three patients had viral re-activations seen on routine monitoring but no signs of clinical disease. Specifically, two patients had asymptomatic CMV reactivation and one patient had adenovirus, all treated successfully with pre-emptive anti-viral therapy. One patient developed transplant associated thrombotic microangiopathy (TA-TMA) on Day +16 and resolved with medical management. The TA-TMA was likely secondary to the calcineurin inhibitor.

One patient had stage 3 skin acute GVHD (overall grade II), 46 days post-transplant, which quickly responded to methylprednisone and then an oral prednisone taper. One patient had both stage 3 skin and stage 1 gastrointestinal (GI) acute GVHD (overall grade II) 40 days post-transplant which responded to methylprednisone and restarting tacrolimus which were subsequently weaned. Two patients had mild chronic skin GVHD.

At a median follow up of 1.1 years (range: 214 days- 1.7 years), all four patients are alive with stable engraftment and full donor chimerism. They have had no evidence of disease and a performance score of 100 at last follow up. Three patients are off immunosuppressive therapy (patient 1 at 18 months, Patient 2 at 130 days, Patient 4 at 247 days), one patient is on gradual wean of immunosuppression for mild chronic skin GVHD.

\section{DISCUSSION}

Following the success of haplo-HCT, in both malignant and non-malignant disorders in recent years, multiple variations to conditioning intensity and GVHD prophylaxis have been employed to improve the outcome even further.

The use of PTCy in haplo-HCT, either with BM or PBSC grafts, has been proven to be safe and associated with low incidence of GVHD in adults $(1,20-22)$. Although promising results have been reported with the use of PTCy in haplo-HCT in a few pediatric studies published to date (23-25), an alternative regimen may be necessary in children with recent prior exposure to high dose cyclophosphamide, those with a contraindication to the use of alkylating agents, organ dysfunction and possibly in younger children $(<10$ years) due to the reported unacceptable higher rate of acute GVHD (aGVHD) and early NRM (26).

Abatacept has emerged as an attractive alternative since a reduction of aGVHD was demonstrated in its first in-human trial with the addition of four peri-transplant doses to standard GVHD prophylaxis in patients with malignant diseases receiving matched unrelated donor (MUD) graft (17).

Aba2, a phase II trial, enrolled patients older than 18 years of age with hematological malignancies, to study the addition of abatacept to calcineurin inhibitor (CNI)/MTX-based GVHD prophylaxis to reduce aGVHD. In 8/8 HLA-MUD, grade III-IV GVHD was $6.8 \%$ in the abatacept group vs $14.8 \%$ in the group. Severe aGVHD free survival (SGFS) was $93.2 \%$ (CNI/MTX plus abatacept) versus $82 \%$ (CNI/MTX plus placebo, $\mathrm{P}=0.05)$. The addition of abatacept did not increase relapse in the $7 / 8$ or $8 / 8 \mathrm{MUD}$ recipients ( $7 / 8 \mathrm{~s} \mathrm{HR}, 0.45 ; \mathrm{P}=0.21$ and $8 / 8 \mathrm{~s} \mathrm{HR}, 0.86 ; \mathrm{P}=0.66)$, a key safety outcome when adding an adjunctive immunomodulating agent to transplants for hematologic malignancies. There was a significant improvement demonstrated in survival indicators in the 7/8 HLA-MUD cohort as well (27). A phase II trial comparing cyclophosphamide and abatacept with standard of care treatment in haematological malignancies is currently recruiting (NCT03680092) 
This approach has since been extended to patients with non-malignant diseases. There has been encouraging results with the use of abatacept in haemoglobinopathies, where unlike malignant disorders, the aim is not to produce a graft versus leukemia effect. Ngwube et al recently published results of a phase 1 trial (NCT03128996) of MUD HCT in patients with severe sickle cell disease (SCD) using reduced intensity conditioning followed by tacrolimus, methotrexate or MMF and abatacept as GVHD prophylaxis (28). The continued risk of cGVHD in the first 2 participants in this trial prompted an amendment to extend costimulation blockade with abatacept to 1 year posttransplant in SCD patients receiving bone marrow product. The incidence of grades III-IV aGVHD at day +100 was $7 \%$ with a 2 -year overall and disease-free survival was $100 \%$ and $92.9 \%$, respectively. One-year incidence of chronic GVHD was $57 \%$ and mild/limited in all but 1 patient who received abatacept for a longer duration. Phase 2 trial of unrelated donor HCT, adding abatacept to standard GVHD prophylaxis are underway.

Khandelwal et al built on their previous experience of using a myeloablative regimen on 24 children with transfusion dependent thalassemia with the addition of four doses of abatacept to CNI and corticosteroids. They also found that abatacept reduced the incidence of aGVHD (No grade II-IV aGVHD in the abatacept cohort vs $50 \%$ in standard cohort) without impacting engraftment or survival (29). All of the above studies were in the unrelated donor setting.

A pilot study by Jaiswal et al, trialed the concept of extended $\mathrm{T}$ cell co-stimulation blockade (COSBL) with Abatacept until day +180 to achieve long term tolerance and decrease incidence of cGVHD. This was administered along with sirolimus and PTCy in 10 patients with severe aplastic anemia. The GVHD and disease-free survival at one year in the COSBL group was $80 \%$ vs. $30 \%$ in the control group $(p=0.05)$. This protocol did result in reduced cGVHD, low incidence of CMV and better immunosuppression free survival at one year (30). Another trial is exploring the benefits of intermediate duration abatacept on the risk of GVHD in sickle cell disease HCT (31). The ideal dosing schedule for abatacept to yield the optimal GVHD free relapse free survival (GRFS) in different disorders is still being investigated.

Another reported application of abatacept pertained to its effects on Natural killer (NK) cells. NK resistance to CTLA4Ig mediated anergy has been shown in both murine and canine models $(32,33)$. NK cells were also found to have augmented anti-tumor effect in the presence of CTLA4Ig (34). Jaiswal et al reported the use of early and sequential CTLA4 (Abatacept) primed DLI starting at day +7 of PTCy based haplo-HCT in 30 patients with relapsed/refractory leukemia. Prophylactic use of DLIs had been successfully used in the setting of advanced haematological malignancies to attenuate the possibility of disease progression (DP). This was based on the principle that NK cell mediated anti-leukemia effect could be exploited without an increase in $\mathrm{T}$ cell-mediated alloreactivity. They showed promising results with an incidence of aGVHD of only $6.7 \%$, limited cGVHD in $20.8 \%$, NRM $4.5 \%$ and a progression free survival (PFS) of $75.8 \%$ (35). This is particularly striking as the reported PFS of 372 patients with high disease risk index after PTCy based haplo-HCT was reported to be $22 \%$ by the Baltimore group (36).

Abatacept represents a unique approach to immunomodulation where prevention of early severe aGVHD is not associated with a delay in overall immune reconstitution. Patients treated with abatacept have been shown to accumulate significantly fewer proliferating and activated $\mathrm{CD} 4^{+} \mathrm{T}$ cells with a reduction in effector memory CD4 T cell expansion, early after transplantation $(17,27)$. That effect was not similarly demonstrated in $\mathrm{CD}^{+}$function and therefore combining $\mathrm{T}$ cell blockade with another signaling pathway may be necessary to control CD 4 and CD8 alloreactivity $(37,38)$. Similar to other studies, reconstitution of NK cells occurred more rapidly compared to other lymphocyte subsets. In the phase 1 trial (NCT01917708), no differences were seen in granulocyte or B cell recovery in non-malignant disease patients compared to Aba2 groups (Aba2; NCT01743131). Patients treated with abatacept have experienced transient T cell and NK depletion, where similar counts are seen by day +100 , and therefore may be less likely to experience $\mathrm{T}$ cell dependent immunocompromise (28).

An additional vital aspect with the introduction of new immunomodulating agents is the added risk of infectious complications. There is in vitro evidence that T cells exposed to CTLA4Ig and cyclosporine still retain virus specific immunity (39). The use of abatacept in patients with severe aplastic anemia resulted 
in lower incidence of CMV and other viral infections which may be explained by the relative lack of effect of abatacept on anti-viral memory $\mathrm{T}$ cells as well as the demonstrated improved recovery of Tregs (30). Ngwube et al also showed $\mathrm{T}$ cell subsets gradually recovered after day +100 to the normal range by 1 year. Although the rates of viral reactivations are non-negligible, majority of the patients in the previous studies were asymptomatic, and treated successfully with antiviral therapy. No incidence of post-transplant lymphoproliferative disorder (PTLD) was reported in the aforementioned studies. Careful routine monitoring for viral activation and immune recovery is needed post-transplant to guide interventions.

Although our study has a small cohort of four patients, it is the only pediatric report to describe outcomes with abatacept in the unique setting where administration of PTCy may be contraindicated. Our report highlights the concept of achieving acceptable GVHD rates with the addition of abatacept to GVHD prophylaxis without compromising the risk of relapse or increasing infectious complications.

\section{CONCLUSION}

Successful Haplo-HCT utilizing an Aba- based regimen can result in reliable engraftment and acceptable GVHD. Similar to other studies, this supports the concept of Aba-induced immune tolerance with minimal treatment-related morbidity. Our small sample size limits generalizability and a prospective study incorporating abatacept as upfront GVHD prophylaxis to validate these results in the haplo-HCT setting is being developed at our program. Alternatives to the PTCy regimen may be especially needed to improve outcomes for patients requiring high risk transplants, such as those undergoing a second HCT or have had significant previous toxicity.

\section{Acknowledgment: None}

Declaration of Interests: The authors declare no potential interests.

\section{References:}

1. Luznik L, O'Donnell PV, Symons HJ, Chen AR, Leffell MS, Zahurak M, et al. HLA-haploidentical bone marrow transplantation for hematologic malignancies using nonmyeloablative conditioning and high-dose, posttransplantation cyclophosphamide. Biology of Blood and Marrow Transplantation. 2008;14(6):641-50.

2. Al Malki MM, Yang D, Labopin M, Afanasyev B, Angelucci E, Bashey A, et al. Comparing transplant outcomes in ALL patients after haploidentical with PTCy or matched unrelated donor transplantation. Blood advances. 2020;4(9):2073-83.

3. Law AD, Salas MQ, Lam W, Michelis FV, Thyagu S, Lipton JH, et al. Reduced-intensity conditioning and dual $\mathrm{T}$ lymphocyte suppression with antithymocyte globulin and post-transplant cyclophosphamide as graft-versus-host disease prophylaxis in haploidentical hematopoietic stem cell transplants for hematological malignancies. Biology of Blood and Marrow Transplantation. 2018;24(11):2259-64.

4. Wang Y, Wu D-P, Liu Q-F, Xu L-P, Liu K-Y, Zhang X-H, et al. Low-dose post-transplant cyclophosphamide and anti-thymocyte globulin as an effective strategy for GVHD prevention in haploidentical patients. Journal of hematology \& oncology. 2019;12(1):1-9.

5. Xu X, Yang J, Cai Y, Li S, Niu J, Zhou K, et al. Low dose anti-thymocyte globulin with low dose posttransplant cyclophosphamide (low dose ATG/PTCy) can reduce the risk of graft-versus-host disease as compared with standard-dose anti-thymocyte globulin in haploidentical peripheral hematopoietic stem cell transplantation combined with unrelated cord blood. Bone marrow transplantation. 2021;56(3):705-8.

6. Makanga DR, Guillaume T, Willem C, Legrand N, Gagne K, Cesbron A, et al. Posttransplant Cyclophosphamide and Antithymocyte Globulin versus Posttransplant Cyclophosphamide as Graft-versus-Host Disease Prophylaxis for Peripheral Blood Stem Cell Haploidentical Transplants: Comparison of T Cell and NK Effector Reconstitution. The Journal of Immunology. 2020;205(5):1441-8.

7. Passweg J, Baldomero H, Bader P, Bonini C, Duarte R, Dufour C, et al. Use of haploidentical stem cell transplantation continues to increase: the 2015 European Society for Blood and Marrow Transplant activity 
survey report. Bone marrow transplantation. 2017;52(6):811-7.

8. Phelan R, Arora, M., Chen, M. . Current use and outcome of hematopoietic stem cell transplantation: CIBMTR US summary slides. 2020.

9. Bhushan V, Mathews J, Vance E, Escobar C, Kurre J, Potter J, et al. Salvage Therapy for Graft Rejection with Second Haploidentical Allogeneic Stem Cell Transplantation (Haplo-HCT) from a Second Related Donor. Biology of Blood and Marrow Transplantation. 2015;21(2):S297.

10. Prata PH, Resche-Rigon M, Blaise D, Socié G, Rohrlich P-S, Milpied N, et al. Outcomes of salvage haploidentical transplant with post-transplant cyclophosphamide for rescuing graft failure patients: a report on behalf of the francophone society of bone marrow transplantation and cellular therapy. Biology of Blood and Marrow Transplantation. 2019;25(9):1798-802.

11. Yoshihara S, Ikegame K, Taniguchi K, Kaida K, Kim E, Nakata J, et al. Salvage haploidentical transplantation for graft failure using reduced-intensity conditioning. Bone marrow transplantation. 2012;47(3):369-73.

12. Epperla N, Pasquini M, Pierce K, Drobyski W, Rizzo J, Horowitz M, et al. Salvage haploidentical hematopoietic cell transplantation for graft rejection following a prior haploidentical allograft. Bone marrow transplantation. 2017;52(1):147-50.

13. Giammarco S, Raiola AM, Di Grazia C, Bregante S, Gualandi F, Varaldo R, Chiusolo P, Sora F, Sica S, Laurenti L, Metafuni E. Second haploidentical stem cell transplantation for primary graft failure. Bone Marrow Transplantation. 2020 Dec 16:1-6.

14. Moreland L, Bate G, Kirkpatrick P. Abatacept. Nature Reviews Drug Discovery. 2006;5(3):185-6.

15. Zhu Z, Fan L, Wang Q. Simultaneous blockade of costimulatory signals CD28-CD80 and CD40-CD154 combined with monoclonal antibody against CD25 induced a stable chimerism and tolerance without graftversus-host disease in rat. European Surgical Research. 2011;46(3):109-17.

16. Tiwari D, Horan J, Langston A, Qayed M, Carr J, Renfroe H, et al. A first-in-disease trial of in vivo costimulation blockade for acute GvHD prevention: the addition of abatacept to standard GvHD prophylaxis controls early CD4+ T cell proliferation and is associated with low rates of severe acute GvHD. American Society of Hematology; 2012.

17. Koura DT, Horan JT, Langston AA, Qayed M, Mehta A, Khoury HJ, et al. In vivo T cell costimulation blockade with abatacept for acute graft-versus-host disease prevention: a first-in-disease trial. Biology of Blood and Marrow Transplantation. 2013;19(11):1638-49.

18. Jagasia MH, Greinix HT, Arora M, Williams KM, Wolff D, Cowen EW, et al. National Institutes of Health consensus development project on criteria for clinical trials in chronic graft-versus-host disease: I. The 2014 diagnosis and staging working group report. Biology of Blood and Marrow Transplantation. 2015;21(3):389-401. e1.

19. Przepiorka D, Weisdorf D, Martin P, Klingemann H, Beatty P, Hows J, et al. 1994 Consensus conference on acute GVHD grading. Bone marrow transplantation. 1995;15(6):825-8.

20. Raiola AM, Dominietto A, di Grazia C, Lamparelli T, Gualandi F, Ibatici A, et al. Unmanipulated haploidentical transplants compared with other alternative donors and matched sibling grafts. Biology of Blood and Marrow Transplantation. 2014;20(10):1573-9.

21. Raj K, Pagliuca A, Bradstock K, Noriega V, Potter V, Streetly M, et al. Peripheral blood hematopoietic stem cells for transplantation of hematological diseases from related, haploidentical donors after reducedintensity conditioning. Biology of Blood and Marrow Transplantation. 2014;20(6):890-5.

22. Solomon SR, Solh M, Morris LE, Holland HK, Bashey A. Myeloablative conditioning with PBSC grafts for $\mathrm{T}$ cell-replete haploidentical donor transplantation using posttransplant cyclophosphamide. Advances in hematology. 2016;2016. 
23. Sharma A, Rastogi N, Chatterjee G, Kapoor R, Nivargi S, Yadav SP. Haploidentical Stem Cell Transplantation With Posttransplant Cyclophosphamide for Pediatric Acute Leukemia is Safe and Effective. Journal of pediatric hematology/oncology. 2020.

24. Pérez-Martinez A, Ferreras C, Pascual A, Gonzalez-Vicent M, Alonso L, Badell I, et al. Haploidentical transplantation in high-risk pediatric leukemia: A retrospective comparative analysis on behalf of the Spanish working Group for bone marrow transplantation in children (GETMON) and the Spanish Grupo for hematopoietic transplantation (GETH). American journal of hematology. 2020;95(1):28-37.

25. Katsanis E, Sapp LN, Reid SC, Reddivalla N, Stea B. T-Cell replete myeloablative haploidentical bone marrow transplantation is an effective option for pediatric and young adult patients with high-risk hematologic malignancies. Frontiers in Pediatrics. 2020;8.

26. Jaiswal SR, Chakrabarti A, Chatterjee S, Ray K, Chakrabarti S. Haploidentical transplantation in children with unmanipulated peripheral blood stem cell graft: The need to look beyond post-transplantation cyclophosphamide in younger children. Pediatric transplantation. 2016;20(5):675-82.

27. Watkins B, Qayed M, McCracken C, Bratrude B, Betz K, Suessmuth Y, et al. Phase II Trial of Costimulation Blockade With Abatacept for Prevention of Acute GVHD. Journal of Clinical Oncology. 2021:JCO. 20.01086.

28. Ngwube A, Shah N, Godder K, Jacobsohn D, Hulbert ML, Shenoy S. Abatacept is effective as GVHD prophylaxis in unrelated donor stem cell transplantation for children with severe sickle cell disease. Blood Advances. 2020;4(16):3894-9.

29. Khandelwal P, Yeh RF, Yu L, Lane A, Dandoy CE, El-Bietar J, et al. Graft Versus Host Disease Prophylaxis With Abatacept Reduces Severe Acute Graft Versus Host Disease in Allogeneic Hematopoietic Stem Cell Transplant for Beta Thalassemia Major with Busulfan, Fludarabine, and Thiotepa. Transplantation. 2020 .

30. Jaiswal SR, Bhakuni P, Zaman S, Bansal S, Bharadwaj P, Bhargava S, et al. T cell costimulation blockade promotes transplantation tolerance in combination with sirolimus and post-transplantation cyclophosphamide for haploidentical transplantation in children with severe aplastic anemia. Transplant immunology. 2017;43:54-9.

31. Chaudhury S, Laskowski J, Rangarajan HG, Abraham A, Haight AE, Guilcher GM, et al. Abatacept for GVHD prophylaxis after hematopoietic stem cell transplantation (HCT) for pediatric sickle cell disease (SCD): a sickle transplant alliance for research (STAR) trial. Biology of Blood and Marrow Transplantation. 2018;24(3):S91.

32. Chen Y, Fukuda T, Thakar MS, Kornblit BT, Storer BE, Santos EB, et al. Immunomodulatory effects induced by cytotoxic T lymphocyte antigen 4 immunoglobulin with donor peripheral blood mononuclear cell infusion in canine major histocompatibility complex-haplo-identical non-myeloablative hematopoietic cell transplantation. Cytotherapy. 2011;13(10):1269-80.

33. Kean L, Hamby K, Koehn B, Lee E, Coley S, Stempora L, et al. NK cells mediate costimulation blockade-resistant rejection of allogeneic stem cells during nonmyeloablative transplantation. American journal of transplantation. 2006;6(2):292-304.

34. Peng Y, Luo G, Zhou J, Wang X, Hu J, Cui Y, et al. CD86 is an activation receptor for NK cell cytotoxicity against tumor cells. PLoS One. 2013;8(12):e83913.

35. Jaiswal SR, Bhakuni P, Joy A, Kaushal S, Chakrabarti A, Chakrabarti S. CTLA4Ig primed donor lymphocyte infusion: a novel approach to immunotherapy after haploidentical transplantation for advanced leukemia. Biology of Blood and Marrow Transplantation. 2019;25(4):673-82.

36. McCurdy SR, Kanakry JA, Showel MM, Tsai HL, Bolanos-Meade J, Rosner GL, et al. Risk-stratified outcomes of nonmyeloablative HLA-haploidentical BMT with high-dose posttransplantation cyclophosphamide. 
Blood. 2015;125(19):3024-31.

37. Khandelwal P, Chaturvedi V, Owsley E, Lane A, Heyenbruch D, Lutzko CM, et al. CD38(bright)CD8(+) T Cells Associated with the Development of Acute GVHD Are Activated, Proliferating, and Cytotoxic Trafficking Cells. Biology of blood and marrow transplantation : journal of the American Society for Blood and Marrow Transplantation. 2020;26(1):1-6.

38. Grogan BM, Tabellini L, Storer B, Bumgarner TE, Astigarraga CC, Flowers ME, et al. Activation and expansion of CD8+ T effector cells in patients with chronic graft-versus-host disease. Biology of Blood and Marrow Transplantation. 2011;17(8):1121-32.

39. Comoli P, Locatelli F, Moretta A, Montagna D, Calcaterra V, Cometa A, et al. Human alloantigenspecific anergic cells induced by a combination of CTLA4-Ig and CsA maintain anti-leukemia and anti-viral cytotoxic responses. Bone marrow transplantation. 2001;27(12):1263-73.

\section{Abbreviations}

\begin{tabular}{llll}
\hline Haplo & Haploidentical & HSV & Herpes Simplex Virus \\
\hline HCT & Hematopoietic Stem Cell Transplant & PCR & Polymerase Chain Reaction \\
GVHD & Graft-Versus-Host-Disease & EBV & Epstein Barr Virus \\
ATG & Anti Thymocyte Globulin & HHV-6 & Human Herpes Virus-6 \\
PTCy & Post Transplant Cyclophosphamide & RBC & Red Blood Cell \\
Aba & Abatacept & CML & Chronic Myeloid Leukemia \\
CMV & Cytomegalovirus & AML & Acute Myeloid Leukemia \\
HLA & Human Leukocyte Antigen & CR & Complete Remission \\
TCD & T cell depletion & MMF & Mycophenolate Mofetil \\
NRM & Non Relapse Mortality & IFGR1 & Interferon Gamma Receptor 1 \\
OS & Overall Survival & IVIG & Intravenous Immunoglobulin \\
GFRS & Graft versus host disease free relapse free survival & AKI & Acute Kidney Injury \\
MUD & Matched Unrelated Donor & CNI & Calcineurin Inhibitor \\
CTLA-4 & Cytotoxic T lymphocyte associated antigen-4 & TDT & Transfusion Dependent Thalassemia \\
PBSC & Peripheral Blood Stem Cells & Bu & Busulfan \\
HEPA & High Efficiency Particulate Air & TNC & Total Nucleated Cells \\
\hline
\end{tabular}

\section{Hosted file}

Table 1- Abatacept word doc.docx available at https://authorea.com/users/422076/articles/

527837-abatacept-based-graft-versus-host-disease-prophylaxis-in-haplo-identical-

hematopoietic-cell-transplant-single-center-experience-in-a-high-risk-cohort 